\title{
The role of surgery in optic pathway/hypothalamic gliomas in children
}

\author{
Clinical article
}

\author{
John Goodden, F.R.C.S.(Neuro.Surg), ${ }^{1}$ Barky Pizer, F.R.C.P.C.H., Ph.D., ${ }^{2}$ \\ Benedetta Pettorini, M.D., ${ }^{1}$ Dawn Williams, M.Sc., R.N., ${ }^{1}$ \\ Jo Blair, M.D., M.R.C.P.C.H., ${ }^{3}$ MoHaMmed Didi, M.R.C.P., ${ }^{3}$ \\ Nicky ThORP, M.R.C.P., F.R.C.R., ${ }^{4}$ AND Conor MALluCCI, F.R.C.S.(SN) ${ }^{1}$
}

Departments of ${ }^{1}$ Pediatric Neurosurgery, ${ }^{2}$ Pediatric Oncology, and ${ }^{3}$ Pediatric Endocrinology, Alder Hey Children's NHS Foundation Trust, Liverpool; and ${ }^{4}$ Department of Radiotherapy, Clatterbridge Cancer Centre NHS Foundation Trust, Bebington, Wirral, United Kingdom

Object. Optic pathway/hypothalamic gliomas (OPHGs) are generally benign tumors situated in an exquisitely sensitive brain region. The location and natural history of OPHGs has led to much debate about optimal treatment. This paper revisits the role of and optimal timing of debulking surgery in OPHG.

Methods. This paper presents a series of cases managed by the neuro-oncology team at Alder Hey Children's Hospital and a single surgeon. Data were collected retrospectively for periods prior to 2009 and prospectively thereafter. Tailored treatment strategies were used, including observation and combinations of surgery, chemotherapy, and radiotherapy. Tumor control rates and outcomes are reviewed.

Results. Forty-two patients were treated between 1998 and 2011. Their median age at diagnosis was 5 years 7 months. Nineteen patients were positive for neurofibromatosis Type 1 (NF1) and 23 patients were negative for NF1. The median duration of follow-up was 77 months (range 21.8-142.3 months). Presenting symptoms included visual impairment (in $50 \%$ of cases), headache (in $24 \%$ ), and hypothalamic/pituitary dysfunction (in $29 \%$ ).

Twenty-two debulking procedures were performed in 21 patients. Four biopsies ( 3 open, 1 endoscopic) were also performed. The histological diagnosis was pilocytic astrocytoma in 21 patients and pilomyxoid astrocytoma in 2 patients. Ten patients (Group 1) had primary surgical debulking alone and were then observed. Four patients (Group 2) had surgical debulking, plus planned chemotherapy within 3 months. Seven patients (Group 3) required surgical debulking for progressive disease following a variety of treatments. Patient age had the greatest impact on subsequent tumor progression.

In total, 13 patients received chemotherapy, 4 on initial presentation, 4 in combination with surgery, and 5 for further tumor progression. Five patients were treated with radiotherapy, 3 prior to referral to Alder Hey.

Eleven patients required shunt insertion for hydrocephalus. Vision was stabilized for $74 \%$ of patients. The number of patients with hypothalamic/pituitary dysfunction increased from 12 at presentation to 16 by the end of treatment. The overall survival rate was $93 \%$. Three patients died -1 from tumor progression, 1 from infective complications from tumor biopsy, and 1 from a spontaneous posterior fossa hemorrhage. NF1 was associated with improved outcome - fewer patients required active intervention and rates of visual impairment and/or or hypothalamic/pituitary dysfunction were lower.

Conclusions. Good long-term survival and functional outcomes can be achieved in children with OPHG. Tumor control was achieved through an individualized approach using surgery, chemotherapy, or radiotherapy in varied combinations. The authors aim to limit radiotherapy to cases involving older children in whom other therapies have failed, due to the well-described and often devastating late effects associated with midline cranial irradiation. Surgery has a clear role for diagnosis, tumor control, and relief of mass effect. In particular, primary surgical debulking of tumor (without adjuvant therapy) is safe and effective. Recent advances in intraoperative MRI may add value and need further assessment.

(http://thejns.org/doi/abs/10.3171/2013.8.PEDS12546)

$\begin{array}{lll}\text { KEY WORDS } & \bullet \text { pediatric neurosurgery } \bullet \text { chiasmatic } \bullet \text { hypothalamic } \\ \text { glioma } \bullet & \text { intraoperative MRI } \bullet \text { oncology }\end{array}$

Abbreviations used in this paper: $\mathrm{BMI}=$ body mass index; $\mathrm{LGG}$ = low-grade glioma; NF1 = neurofibromatosis Type $1 ;$ OPHG = optic pathway/hypothalamic glioma; SIOP = International Society of Paediatric Oncology; VP = ventriculoperitoneal.
$\mathrm{O}$ PTIC pathway/hypothalamic gliomas (OPHGs) are a relatively rare tumor type. They represent $2 \%-7 \%$ of all pediatric intracranial tumors, with $65 \%$ of these lesions presenting in children younger than 5 years of age. . $^{, 17,21,26,31,38}$ Tumor presentation is often due 


\section{J. Goodden et al.}

to pressure on or compression of the optic pathways and diencephalon. Some patients may also present with hydrocephalus from tumor blocking CSF pathways. ${ }^{2,26}$

These tumors are nearly always histologically lowgrade tumors. The majority are pilocytic astrocytomas (WHO Grade I), although a proportion will be pilomyxoid astrocytomas and rarely WHO Grade II neoplasms. ${ }^{6,8,12}$ $17,23,25,35$ A significant proportion $(10 \%-70 \%)$ occur in patients with neurofibromatosis Type 1 (NF1). ${ }^{7}, 14,17,18,23,26,29,31,41$

Up to half of OPHGs are reported to remain stable and not require intervention; hence an initial watch-andwait policy is often employed. This is particularly the case if there is no imminent threat to vital functions and also in patients with NF1. Much of the published literature currently focuses on the management of these tumors with observation, chemotherapy, or radiotherapy, whereas there is a paucity of literature on neurosurgery for these tumors and a lack of consensus regarding its place in multimodal therapy. ${ }^{2,21,29,47}$

Historically, there has been a reluctance to support the role of surgery for OPHGs, largely due to their histologically benign nature, their central location, and their relationship to the visual pathway.

A recently published paper reports the results of a multidisciplinary consensus conference that addressed the surgical treatment of these lesions. The authors write: "It was agreed that primary attempted surgical resection of HCLGG [hypothalamic chiasmatic low-grade glioma] is not the current recommended standard of care. However, it was also acknowledged that selected cases were amenable to attempted resection or debulking with low risk." ${ }^{46}$

In the present paper, we seek to add to the discussion about the role of surgery both in terms of early intervention in some selected cases and as therapy in other progressive cases. Within this series, the major surgical interventions were performed by a single surgeon (C.M.).

\section{Methods}

Data were collected retrospectively for periods prior to 2009 and prospectively between 2009 and 2011. Case notes pertaining to patients identified from the neurosurgery and neuro-oncology departmental databases were reviewed in detail.

Data were collected regarding presenting symptoms, visual function, endocrine function, NF1 status, tumor size and location, neuroradiological imaging characteristics, histopathological findings, treatment (surgery, chemotherapy, and radiotherapy), and control/progression rates. Two cohorts were identified: patients with NF1 (NF1 cohort) and those without NF1 (non-NF1 cohort).

Patients were included in this study if they had tumors involving the optic chiasm and/or hypothalamus (Dodge types B and C, modified Dodge types 2, 3, and 4). ${ }^{43}$ Pure optic nerve tumors without chiasmatic involvement (Dodge Type A, modified Dodge Type 1) have been excluded from this study, as none of the patients in our institution have required surgery for these.

Seven patients were referred for a second opinion after receiving prior treatment (including chemotherapy, radiotherapy, and/or limited surgery) in their local center.
Treatment strategies included observation and combinations of chemotherapy, surgery (biopsy or debulking), and radiotherapy. Due to the complex nature of OPHGs and their treatment, many of our patients have had a number of defined epochs of treatment. In these situations, symptomatic and/or radiological tumor progression typically led to intervention (surgery or chemotherapy), which then resulted in a further period of tumor stability and therefore observation. This cycle would be repeated as required, with intervention tailored to the patient's requirements on each occasion.

The extent of resection was divided into 4 categories: biopsy, $<50 \%$ tumor debulking, 50\%-70\% tumor debulking, and $>70 \%$ tumor debulking. Due to the eloquent nature of the surrounding structures, we advocated that gross-total resection was not appropriate, as the focus was on preservation of function as well as reduction of tumor bulk.

Care of these children was coordinated through a weekly multidisciplinary neuro-oncology meeting and clinic. Regular ophthalmic assessments were performed with fundoscopy, and visual fields and visual acuity were monitored regularly.

Endocrine status was carefully monitored with further investigation and treatment where indicated. Body mass index (BMI) was calculated by the formula: BMI $=($ weight in $\mathrm{kg}) /(\text { height in } \mathrm{m})^{2}$. This was then converted to standard scores derived from the 1990 growth standards. ${ }^{15}$ Patients with BMI standard scores greater than 1.33 (91st percentile) were classified as overweight and those with BMI standard scores greater than 2.00 (98th percentile) were classified as obese.

\section{Results}

\section{Patient Demographics}

Forty-two patients with OPHG were identified as having received treatment at Alder Hey Children's Hospital between 1998 and 2011. Nineteen (45\%) of these 42 patients had NF1. For the whole group, the median age at diagnosis was 5 years 7 months (range 13 months to 16 years 8 months, mean 7 years 3 months). When patients with and without NF1 are considered separately, the median age at presentation was 7 years 2 months for the nonNF1 cohort and 4 years 7 months for the NF1 cohort.

The numbers of male and female patients in the overall group were almost equal (20 male, 22 female). However, when the non-NF1 and NF1 cohorts were separated, opposing sex trends were noted-in the non-NF1 cohort $39 \%$ of the patients were male, $61 \%$ female; in the NF1 cohort, $58 \%$ were male, $42 \%$ female.

\section{Clinical Presentation}

The clinical presentation was varied. Patients with NF1 have been considered as a separate cohort because they generally had a more benign presentation and course.

Overall, 21 patients $(50 \%)$ presented with visual impairment $-65 \%$ of those in the non-NF1 cohort and $32 \%$ of those in the NF1 cohort. The impairment included impaired color vision, diminished visual acuity, visual field 
defects, and optic atrophy. Ten patients (24\%) had headaches as a result of either tumor mass effect or hydrocephalus $-35 \%$ of those in the non-NF1 cohort and $11 \%$ of those in the NF1 cohort.

Eleven patients overall (26\%) had evidence of hypothalamic or pituitary dysfunction; these patients were all in the non-NF1 cohort and represented $48 \%$ of this group. Of the non-NF1 cohort, 5 patients $(22 \%)$ presented with precocious puberty, and 3 patients $(13 \%)$ had other anterior pituitary dysfunction, 3 patients $(13 \%)$ had diencephalic syndrome. Additionally, 10 patients were obese at presentation (with BMI standard scores $>2.00$ ).

In 13 patients, OPHG was diagnosed incidentally, as a result of routine MRI screening of patients with NF1; these 13 patients represent $68 \%$ of the NF1 cohort. As per accepted guidelines, biopsy was not performed in nonprogressive tumors in patients with NF1 when classical radiological and clinical features were present.

\section{Diagnosis}

The diagnosis of OPHG was initially made on the basis of MRI findings in all 42 patients. Twenty-one patients $(50 \%)$ had debulking surgery, and an additional 2 patients underwent tumor biopsy. The histological diagnosis was pilocytic astrocytoma in 21 of these 23 cases and pilomyxoid astrocytoma in 2 cases. Details of the timing and types of surgery are further discussed in the treatment section below.

\section{Tumor Location}

As stated above, pure optic nerve tumors have been excluded from this study. Tumors included had to involve at least the chiasm or hypothalamus (Fig. 1). A difference in tumor location was noted between the NF1 and nonNF1 cohorts. In patients in the non-NF1 cohort, 22 of 23 tumors involved the hypothalamus ( 5 had purely hypothalamic tumors), with optic nerve/chiasm involvement in 18 of 23 cases. However, in patients in the NF1 cohort, only 3 of 19 tumors involved the hypothalamus, and there was optic nerve and chiasm involvement in all 19.

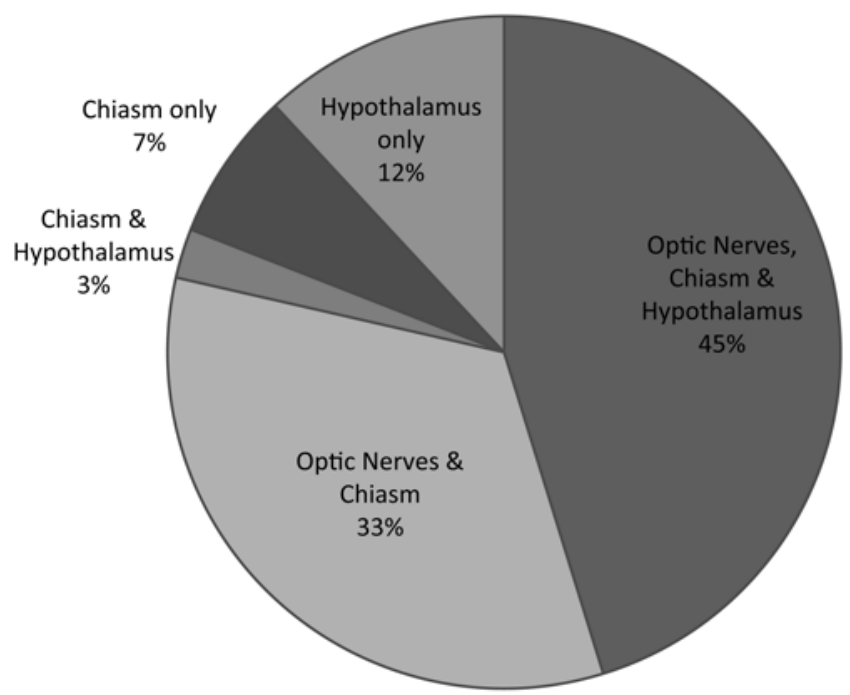

FIG. 1. Tumor location on MRI.

\section{Treatment}

A tailored approach to management was used in all cases, with full multidisciplinary discussion of each case and with choices between observation, chemotherapy, and surgery as initial options.

Indications for a treatment intervention included raised intracranial pressure (from tumor bulk or hydrocephalus), documented visual deterioration, diencephalic syndrome, and tumor progression on serial imaging.

An initial observation strategy was adopted for 21 patients (16 in the NF1 cohort, 5 in the non-NF1 cohort).

The median duration of follow-up for this study was 77 months from the time of initial diagnosis (range 21.8142.3 months), excluding a patient who died 6 weeks after an endoscopic biopsy (see below).

Chemotherapy. In total, 13 patients were treated with chemotherapy. Initial chemotherapy was according to the International Society of Paediatric Oncology (SIOP) low-grade glioma (LGG) trial protocols, LGG-1 or LGG2 (current trial), using a combination of vincristine and carboplatin.

Four patients were treated on initial presentation, with chemotherapy as first-line treatment. Two of these 4 had undergone biopsy prior to treatment, and the findings confirmed the diagnosis of pilocytic astrocytoma in these 2 cases.

Four patients (see surgery Group 2 below) underwent planned initial upfront debulking surgery, followed by chemotherapy as part of their planned treatment. This group was deemed high risk due to their young age (range 1-5 years old), poor visual status, and in 1 case, diencephalic syndrome.

Five patients required chemotherapy following further disease progression at intervals between 2.2 and 35.6 months after surgery. Second-line chemotherapy with vinblastine was successfully used in 2 patients.

Surgery and Surgical Technique. Surgical debulking was conducted in a total of 21 patients (19 in the non-NF1 cohort and 2 in the NF1 cohort) - 15 with newly diagnosed tumors and 6 who had previously been treated at other centers. An additional 2 patients only had biopsies performed (1 endoscopic and 1 via craniotomy). Twentytwo debulking operations were performed in total-13 via midline approaches, 8 via pterional approaches, and 1 via a cerebellopontine angle approach.

The main indication for primary surgical debulking was the presence of a large tumor causing raised intracranial pressure and/or hydrocephalus (in 14 cases). Visual deterioration alone (in the absence of hydrocephalus) was not an indication for surgery except in 1 case in which a cystic portion of tumor was compressing the optic nerves. Surgical debulking had a further role as a delayed intervention for tumor progression after failed chemotherapy and/or radiotherapy (in 7 cases).

The extent of tumor debulking achieved was $>70 \%$ for 16 procedures $(73 \%), 50 \%-70 \%$ for 3 procedures, and $<50 \%$ for 3 procedures.

Patients undergoing resective surgery have been divided into 3 groups (as described in the following para- 
graphs), according to their treatment plan (Fig. 2). Selection for early surgery (Groups 1 and 2, surgery before chemotherapy) was made on the following criteria: 1) the presence of progressive clinical symptoms/signs attributable to tumor (with or without hydrocephalus) at presentation and either 2) a large exophytic tumor growing upward in the midline into the third ventricle causing obstruction or imminent obstruction of the foramina of Monro (treated via a transcallosal route) or 3) a large, clearly exophytic lateral or cystic extension causing mass effect or compression of adjacent structures (treated with pterional or endoscopic cyst drainage).

Patients in Group 1 (10 patients, Table 1) had planned surgical debulking as their main treatment with no adjuvant therapy planned at that stage. For 7 patients, surgery was performed within 2 months of presentation as their planned first treatment. Three patients underwent surgery after their tumors progressed (at 3.4 months, 54.5 months, and 116.7 months). The patients' median age at the time of surgery was 11.7 years (range $2-15.3$ years). In 7 of 10 cases, the patients' condition remained stable following surgery with no other treatment interventions required, with a median follow-up of 66.9 months postoperatively (range 7.3-95.7 months). Progression was seen in 3 cases, at a median interval of 4.3 months postoperatively (range 4-39 months). The progression was treated with chemotherapy (in 1 case), radiotherapy (1 case), and repeat surgery ( 1 case-transcallosal, $70 \%$ debulking). In the patient who had repeat surgery, the tumor progressed again after 16 months and the disease was then stabilized with chemotherapy.

The patients in Group 2 (4 patients, Table 2) under- went planned upfront surgical debulking, followed by adjuvant chemotherapy within 3 months as part of their planned treatment strategy. Their median age at the time of surgery was 3.5 years (range $1.2-5.8$ years). One patient received additional concurrent radiotherapy. All have remained in stable condition, with a median followup of 44.6 months (range 14-66.3 months).

The patients in Group 3, the salvage treatment group (7 patients, Table 3), required debulking surgery for progressive disease, having already undergone a variety of previous treatments, including radiotherapy (1 patient), chemotherapy (4 patients), and chemotherapy plus radiotherapy ( 2 patients). The median age at initial diagnosis for this group was 7.2 years (1.1-13.1 years). At the time of our surgery, the median age was 11.3 years (5.2-16.3 years). For 6 of these patients, surgery was the final stabilizing event, with a median follow-up of 24.3 months (range 10.3-98.3 months). Five patients in this group had been initially treated in other centers and were then referred to us after their tumors had progressed. Three patients had previously had a biopsy (open or stereotactic). Three patients had undergone a previous very limited debulking operation in their local center. One patient had undergone a previous biopsy and cyst drainage in our center to decompress the optic nerves at initial presentation (Fig. 3).

Radiotherapy. Our preference was to keep radiotherapy for progression after failure of other treatments.

Only 5 patients (12\%) in this cohort had radiotherapy. In all 5 cases, it was administered as adjuvant therapy, not as primary treatment. Three patients were treated with radiotherapy prior to referral to Alder Hey, following surgi-

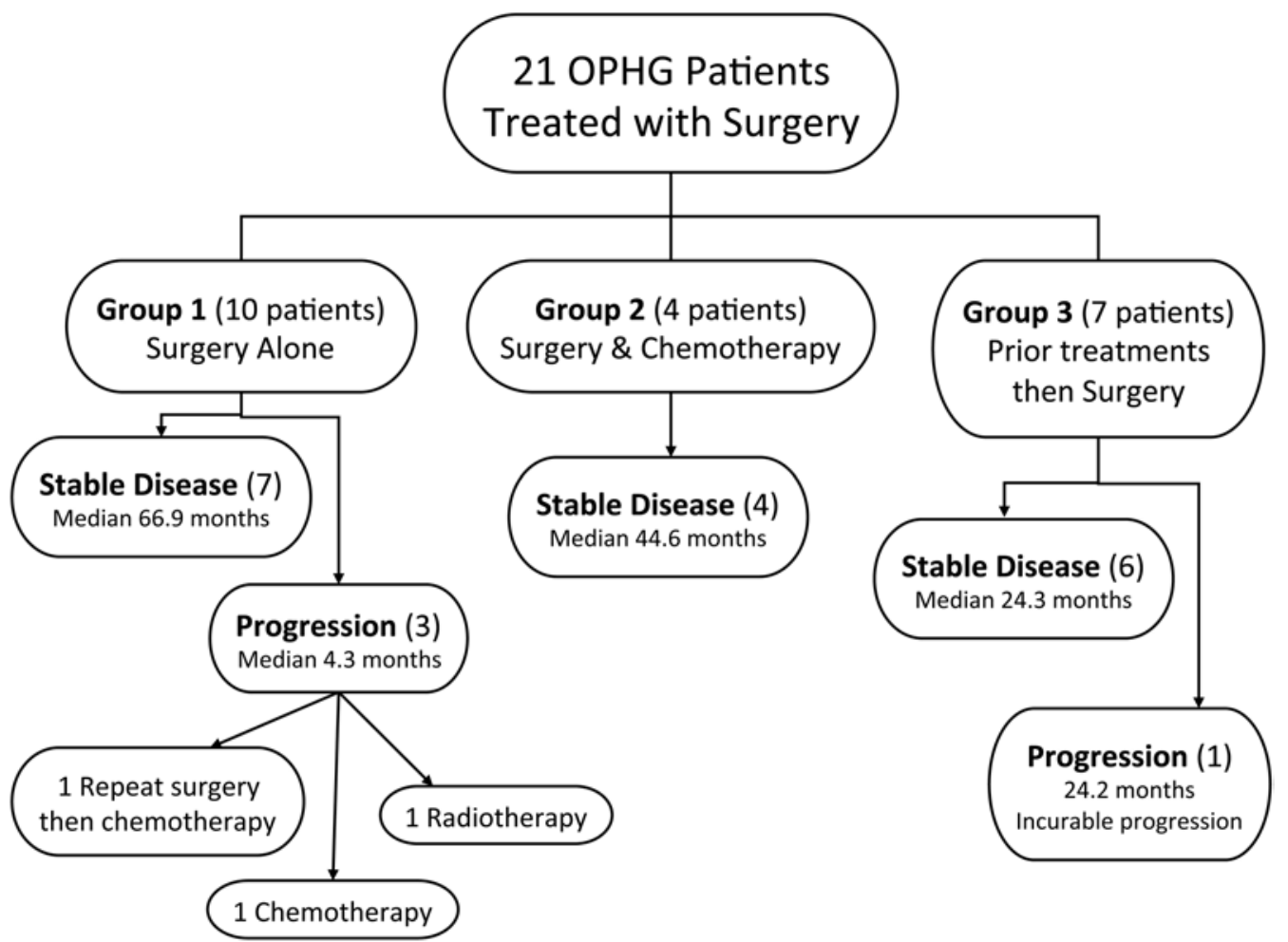

FIG. 2. Summary diagram illustrating outcomes for the 21 patients treated with surgical debulking, according to treatment group. 
Surgery in children with OPHG

TABLE 1: Summary of patient demographic characteristics and treatment for Group 1*

\begin{tabular}{|c|c|c|c|c|c|c|c|c|c|c|c|}
\hline $\begin{array}{l}\text { Age at } \\
\text { Dx (yrs), } \\
\text { Sex }\end{array}$ & $\begin{array}{c}\text { Mo \& Yr } \\
\text { of Dx }\end{array}$ & NF1 & $\begin{array}{c}\text { Mo \& Yr } \\
\text { of Op }\end{array}$ & $\begin{array}{l}\text { Age } \\
\text { at Op } \\
\text { (yrs) }\end{array}$ & $\begin{array}{l}\text { Time From } \\
\text { Presentation } \\
\text { to Op (mos) }\end{array}$ & Op Type & $\begin{array}{c}\% \\
\text { Debulk }\end{array}$ & Hist & $\begin{array}{l}\text { Time Postop } \\
\text { to Progression } \\
\text { (mos) }\end{array}$ & $\begin{array}{l}\text { Length of } \\
\text { of Postop } \\
\text { FU (mos) }\end{array}$ & $\begin{array}{l}\text { Treatment for } \\
\text { Progression }\end{array}$ \\
\hline $3.5, \mathrm{M}$ & Dec '02 & yes & March '03 & 3.8 & 3.4 & pterional & 95 & PA & & 95.7 & \\
\hline $8.7, \mathrm{M}$ & April '02 & no & Oct'06 & 13.2 & 54.5 & transcallosal & 70 & $\mathrm{PA}$ & & 48.2 & \\
\hline $5.5, \mathrm{M}$ & Jan '01 & no & Sept'10 & 15.3 & 116.7 & $\begin{array}{l}\text { midline inter- } \\
\text { hemispheric }\end{array}$ & 70 & PA & & 7.3 & \\
\hline $7.5, \mathrm{~F}$ & Sept '05 & no & Sept '05 & 7.5 & 0.1 & pterional & $<50$ & PA & & 66.9 & \\
\hline $10.4, \mathrm{M}$ & Oct '04 & no & Nov '04 & 10.5 & 0.7 & pterional & 75 & PA & & 76.5 & \\
\hline $12.8, \mathrm{M}$ & April '08 & no & April '08 & 12.8 & 0.1 & transcallosal & 70 & PA & & 35.4 & \\
\hline $15.2, \mathrm{M}$ & Dec'03 & no & Jan '04 & 15.3 & 1.4 & transcallosal & 70 & $\mathrm{PA}$ & & 87.0 & \\
\hline 7.1, M & Feb '08 & no & March '08 & 7.1 & 0.6 & pterional & 80 & PA & 3.7 & & RT \\
\hline $2.0, \mathrm{~F}$ & March '05 & no & March '05 & 2.0 & 0.0 & pterional & 60 & PA & 4.1 & & chemo \\
\hline $14.5, \mathrm{~F}$ & March '02 & no & April '02 & 14.5 & 0.3 & transcallosal & 99 & PA & 38.9 & & $\begin{array}{l}\text { repeat op (transcallosal } \\
70 \% \text { ), later required } \\
\text { chemo }\end{array}$ \\
\hline
\end{tabular}

* Chemo = chemotherapy; Dx = diagnosis; FU = follow-up; Hist = histology; PA = pilocytic astrocytoma; RT = radiotherapy.

cal biopsy or very limited debulking. The other 2 patients required radiotherapy for progressive disease 6 months after surgical debulking at Alder Hey $(80 \%$ debulking in 1 case, $<50 \%$ debulking in 1 case).

The median age at treatment was 7 years 7 months (range 5 years 6 months to 13 years 3 months). The median time to radiotherapy treatment from diagnosis was 6 months (range 2 months to 4 years 3 months).

One of the patients treated prior to referral to Alder Hey had suffered severe complications from radiotherapy, developing moyamoya syndrome and requiring cerebral revascularization

Hydrocephalus. During the course of treatment, 11 patients required CSF diversion for hydrocephalus. These patients were treated by insertion of a ventriculoperitoneal (VP) shunt. Of the 11 shunt-treated patients only one has bilateral shunts, with the remainder having a single shunt. Where possible, bilateral shunts were avoided by performing endoscopic septum pellucidotomy (in 1 case) or open septal fenestration at the time of tumor surgery.

\section{Outcome}

Functional Follow-Up. In this series, 21 patients $(50 \%)$ presented with visual symptoms, with many pa- tients already having severely limited vision. The deficits ranged from impairment of color vision to visual field defects to severely impaired visual acuity and almost complete blindness.

During the time of treatment and monitoring, 9 patients (21\%) had deterioration in vision, but visual status was maintained at the presentation level in 31 patients (74\%). Better visual outcomes were noted for the NF1 cohort; vision remained stable in $84 \%$ of patients in the NF1 cohort and $65 \%$ of the non-NF1 cohort.

Visual deterioration was not always accompanied by an obvious change in the MRI appearance of the tumor, but did always prompt a reassessment MRI scan and reappraisal of the treatment regimen. Patients with deteriorating vision were treated with chemotherapy as the first option. Debulking surgery was not indicated for or used as a treatment for deteriorating vision.

During treatment and follow-up, the number of patients with endocrinopathy increased from 12 (29\%) at presentation to $16(38 \%)$. These patients required a variety of pituitary replacement hormones. Six patients had precocious puberty, although four had presented with this. Two patients required treatment for diabetes insipidus that had developed as a result of treatment.

TABLE 2: Summary of patient demographic characteristics and treatment for Group 2*

\begin{tabular}{|c|c|c|c|c|c|c|c|c|c|c|c|}
\hline $\begin{array}{l}\text { Age (yrs) } \\
\text { at Dx, Sex }\end{array}$ & NF1 & $\begin{array}{c}\text { Mo \& Yr } \\
\text { of Op }\end{array}$ & $\begin{array}{l}\text { Age at } \\
\text { Op (yrs) }\end{array}$ & Op Type & $\begin{array}{c}\% \\
\text { Debulk }\end{array}$ & Hist & $\begin{array}{c}\text { Chemo } \\
\text { Start }\end{array}$ & $\begin{array}{c}\text { Chemo } \\
\text { Regimen }\end{array}$ & $\begin{array}{l}\text { Presentation } \\
\text { to Chemo } \\
\text { (mos) }\end{array}$ & RT & $\begin{array}{l}\text { Length of } \\
\text { FU Post } \\
\text { Tx (mos) }\end{array}$ \\
\hline $4.0, \mathrm{M}$ & no & Nov '05 & 4.0 & pterional & $50-70$ & PA & Dec '05 & LGG2 & 0.8 & & 45.2 \\
\hline $2.9, \mathrm{~F}$ & no & Jan '06 & 3.0 & pterional & $50-70$ & PA & Feb '06 & LGG2 & 0.8 & & 44.0 \\
\hline $1.2, F$ & no & Dec '08 & 1.2 & transcallosal & 70 & PA & Feb '09 & LGG2 & 2.4 & & 14.0 \\
\hline $5.8, F$ & yes & Dec '03 & 5.8 & transcallosal & $<50$ & PMA & Feb '04 & LGG2 & 2.4 & June '04 & 66.3 \\
\hline
\end{tabular}

* PMA = pilomyxoid astrocytoma; $\mathrm{Tx}=$ treatment. 
TABLE 3: Summary of patient demographic characteristics and treatment for Group 3*

\begin{tabular}{|c|c|c|c|c|c|c|c|c|c|c|c|}
\hline $\begin{array}{l}\text { Age at Dx } \\
\text { (yrs), Sex }\end{array}$ & NF1 & Chemo & RT & Op Date & $\begin{array}{l}\text { Age at } \\
\text { Op (yrs) }\end{array}$ & $\begin{array}{l}\text { Presentation } \\
\text { to Op (mos) }\end{array}$ & Op Type & $\begin{array}{c}\% \\
\text { Debulk }\end{array}$ & Hist & $\begin{array}{l}\text { Length } \\
\text { of FU } \\
\text { (mos) }\end{array}$ & $\begin{array}{c}\text { Length } \\
\text { Stable } \\
\text { Postop (mos) }\end{array}$ \\
\hline $9.1, F$ & no & & May '06 & May'10 & 13.5 & 52.6 & CPA approach & 70 & PA & 63.1 & 10.5 \\
\hline $7.2, \mathrm{~F}$ & no & May '98 & & Feb '04 & 13.1 & 70.2 & transcallosal & 90 & PA & 97.0 & 24.2 \\
\hline $3.6, M$ & no & Oct '08 & & May'10 & 5.2 & 19.8 & transcallosal & 40 & PA & 30.1 & 10.3 \\
\hline $3.9, F$ & no & July '03 & & Feb '08 & 11.3 & 87.9 & transcallosal & $>75$ & PMA & 127.0 & 39.0 \\
\hline $1.1, \mathrm{M}$ & no & Aug '02 & Nov '06 & Sept '08 & 7.3 & 74.0 & transcallosal & $>75$ & PA & 106.1 & 32.1 \\
\hline $13.1, \mathrm{~F}$ & no & July '07 & Oct '04 & Oct '07 & 16.3 & 38.5 & pterional & 90 & PA & 78.3 & 24.3 \\
\hline $9.0, F$ & no & April '01 & & Dec '02 & 10.8 & 20.7 & transcallosal & 80 & PA & 119.0 & 98.3 \\
\hline
\end{tabular}

* The values in boldface represent treatments given in other centers before referral for treatment in Alder Hey. CPA $=$ cerebellopontine angle.

Measurements of height and weight at diagnosis were available for 31 patients, of whom 28 also had measurements from their most recent assessment. In 1 patient these measurements were available at the most recent assessment only. At diagnosis, the median BMI standard score was -1.05 (range -2.22 to 3.96), with 4 patients overweight and 10 obese. At the most recent assessment, the median BMI standard score was -0.36 (range -1.11 to 3.60), with 6 patients overweight and 7 obese. Of the 10 patients who were obese at presentation, 3 remained obese at the most recent assessment, and the BMI standard score fell in 7 (4 overweight and 3 normal). Two patients with a normal BMI standard score at diagnosis gained weight to become obese, and 2 patients in the overweight range gained weight, becoming obese.

The proportion of patients with endocrine disturbance was higher in the non-NF1 cohort than in the NF1 cohort. For the non-NF1 cohort, at presentation, 3 patients (13\%) had diencephalic syndrome, and $5(22 \%)$ had precocious puberty. In the NF1 cohort, no patients had diencephalic syndrome or precocious puberty.

Complications of Surgery. No patients had visual deterioration due to surgery. One patient had a neurological deficit with a transient left hemiparesis that improved with postoperative rehabilitation. Two patients had postoperative infections that necessitated a return to theater. There were 3 cases of permanent diabetes insipidus and 2 cases of transient diabetes insipidus.

One patient suffered an intratumoral hemorrhage following endoscopic biopsy and required treatment with external ventricular drainage, which was then complicated by fungal ventriculitis, which then led to the patient's death.

Apart from the above cases of permanent diabetes insipidus (3 cases), there was no case of new attributable hypothalamic injury or endocrinopathy following surgery. There was no case of diabetes insipidus plus adipsia. No patient developed obesity as a direct consequence of surgery.

Survival. Overall survival in this series was $92.9 \%$. During the whole follow-up period (median 6.4 years, range 1.8-11.9 years), 3 patients in this cohort have died.
Only 1 death occurred due to progressive disease, 97 months after initial presentation. This patient's tumor progressed relentlessly, despite chemotherapeutic, surgi$\mathrm{cal}$, and radiotherapeutic interventions over that 8-year period. One patient died of fungal infection following an endoscopic tumor biopsy (as above). The other patient died of a spontaneous fourth-ventricle hemorrhage with otherwise stable disease, 9 years after initial presentation. She presented with a sudden collapse with obstructive hydrocephalus due to this hemorrhage and did not recover following insertion of an external ventricular drain. No obvious cause for the hemorrhage was found.

Effect of NF1. We examined the effects of NF1 status on outcome in this case series. As can be seen in Table 4, our data agree with other series in which the tumors generally follow a more benign course in patients with NF1, with fewer tumors progressing and requiring treatment. In comparison with patients in the non-NF1 cohort, fewer patients in the NF1 cohort suffered deterioration in their vision and fewer were troubled by hypothalamic or pituitary dysfunction.

\section{Discussion}

The majority of OPHGs are low-grade, benign tumors (WHO Grade I or II). ${ }^{8,12,17,23,25,26,35}$ Nevertheless, these tumors present a considerable and often life-long challenge for the neuro-oncology team. A proportion of these tumors behave in a dormant fashion and require no treatment. This is particularly noticeable in patients who have NF1, although it is not exclusively the case. $7,14,17,18,23$, ${ }^{29,41}$ A significant number of tumors will, however, grow and require therapeutic interventions. $23,26,27,29$ These interventions should be carefully planned and timed with the aim of controlling the tumor itself, with the potential for treatment-related morbidity taken into account. ${ }^{19}$

Consensus certainly exists over the range of treatment options that should be considered for these tumorswatch/wait/rescan, chemotherapy, radiotherapy, and surgery. The main emphasis in the last decade has been on chemotherapy as a first-line treatment for symptomatic or progressive tumors, particularly in younger children..$^{21,26}$ 

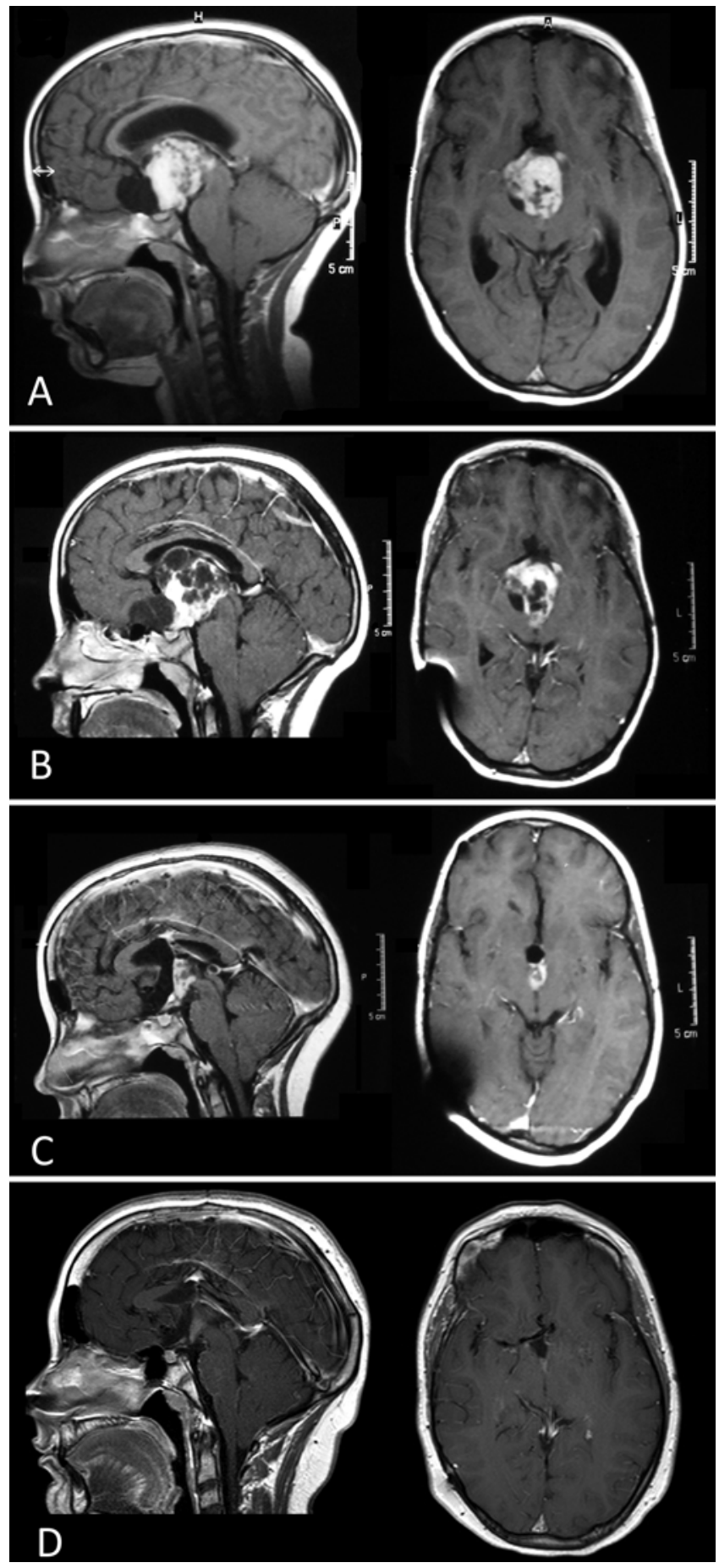

FIG. 3. Serial T1-weighted, Gd-enhanced sagittal and axial MR images obtained in a representative case, demonstrating management over time. This patient first presented in 2001, aged 9 years, with precocious puberty and slow deterioration in vision. Initial MRI scans (A) showed a suprasellar tumor with a cystic portion compressing the optic nerves. The patient underwent a right pterional craniotomy, cyst decompression, and tumor biopsy. Histological examination confirmed the diagnosis of pilocytic astrocytoma. The patient subsequently received chemotherapy, but the tumor progressed after 20 months (B), requiring a midline transcallosal approach and debulking of the lesion. Postoperative MRI (C) showed significant debulking, estimated at about $80 \%$. Subsequent follow-up scans (D) showed further tumor involution despite no active treatment at that stage.
TABLE 4: Comparison of outcomes for patients with and without NF1

\begin{tabular}{lcc}
\hline \multicolumn{1}{c}{ Variable } & $\begin{array}{c}\text { NF1 Cohort } \\
(\mathrm{n}=19)\end{array}$ & $\begin{array}{c}\text { Non-NF1 } \\
\text { Cohort }(\mathrm{n}=23)\end{array}$ \\
\hline progression & $2(11 \%)$ & $24(61 \%)$ \\
chemo & $3(16 \%)$ & $10(43 \%)$ \\
op & $2(11 \%)$ & $21(91 \%)$ \\
RT & $1(5 \%)$ & $4(17 \%)$ \\
stable vision & $16(84 \%)$ & $15(65 \%)$ \\
hypothalamic-pituitary dysfunction & $1(5 \%)$ & $15(65 \%)$ \\
\hline
\end{tabular}

Although it is accepted that radiotherapy can often be effective in controlling growth in these tumors, the late side effects of radiotherapy have led to a reduction in its use, particularly as most of these patients should be expected to survive into adulthood.

There remains controversy over the precise role of neurosurgery, ${ }_{16,18,19,24,35,39-42,47}$ with many teams regarding this as an intervention of last resort with potentially unacceptable morbidity.

At a recent consensus conference, ${ }^{46}$ although surgery was not acknowledged to be the primary treatment modality it was noted:

"Multidisciplinary discussion identifies some patients with characteristics (e.g., cystic tumors, large tumors, and exophytic or hypothalamic tumors) when early surgical debulking is possible with acceptable risk. The proposed anatomical classification will be designed to assist with selection of these cases."

Its was also noted that further international trials were needed to guide treatment strategies for these tumors:

"The selection and testing of surgical and nonsurgical treatments requires an active international trials program, including patients with newly diagnosed cases and patients requiring second- and third-line or subsequent therapies."

In this paper we seek to add to the discussion about the role of neurosurgery for patients with OPHG and to discuss the indications for early versus late surgery as well as preferred routes and approaches. While recognizing the limitations of single-institution cohort studies, we hope that this paper will contribute to future collaborative studies regarding the selection of patients for surgery, the decision making, and the timing of surgical intervention.

\section{Expectant Management-Watch/Wait/Rescan}

A number of patients have tumors that do not need immediate treatment on first presentation. For these patients it is clear that an initial watch/wait/rescan policy should be adopted. These patients include 1) those with small tumors confined to the optic nerves or chiasm, with preserved visual function; and 2) those with small tumors that are not obstructing CSF flow pathways.

As previously indicated, patients with NF1 tend to have a more benign course, with fewer patients requiring treatment for tumor progression and therefore fewer secondary effects from both their tumor and the treatments required over time to control it. 


\section{J. Goodden et al.}

\section{Chemotherapy}

Chemotherapy forms an important part of treatment for OPHG. ${ }^{21,29,32,33,38-40}$ The particular advantages of chemotherapy are that it can be given to younger children with relatively low rates of associated complications and low risks of longer-term late effects. ${ }^{26,29,38}$ The chemotherapy regimens employed for OPHG have changed over the years, but its optimal role and the optimal agents have yet to be clarified. ${ }^{21}$ As in this series, recent chemotherapy treatment regimens have generally been according to clinical trials, such as the SIOP LGG-2 study, which uses a combination of vincristine and carboplatin. Chemotherapy can be the only therapy that a child requires and can prevent the need for radiotherapy or surgery, which is of particular importance in children under 5 years of age. ${ }^{23,29,38-40}$ It is frequently the primary treatment in patients, particularly those of young age, with visual deterioration due to this disease.

\section{Radiotherapy}

The role of radiotherapy for OPHG has been much discussed. While clearly effective, radiotherapy is, however, associated with a number of late effects, including neurocognitive impairment, second-tumor formation, and increasing reports of the occurrence of moyamoya syndrome. ${ }^{2,5,10,11,36,45}$ One of the patients in this series required revascularization surgery for this unusual but potentially devastating complication. Radiotherapy has been also associated with increased rates of endocrine deficit (especially growth hormone deficiency) compared with other treatment modalities. ${ }^{14}$ If radiotherapy is given to younger patients, the neurocognitive consequences are typically more severe and can have a devastating effect on the patient's future life. ${ }^{5}$ Most authors now recommend avoiding radiotherapy in patients under 5 years, and leaving this treatment until as late as possible..$^{5,26,39}$ For these reasons, the rationale behind the management of this cohort of patients has been to avoid upfront radiotherapy as a first-line treatment.

\section{Neurosurgery}

Neurosurgery for OPHG is divided into 2 broad categories: surgery for the secondary effects of the tumor or treatment, and surgery for the tumor itself.

The potential roles of tumor surgery are either for biopsy for diagnostic tissue sampling or for tumor debulking. The role of biopsy in this group has been addressed in the recent consensus conference publication, and our unit has followed this policy. ${ }^{46}$

In general, total resection for low-grade gliomas of the central nervous system is associated with improved 5-year survival rates..$^{12,13,22,41}$ However, we do not consider that gross-total resection of OPHG is a feasible concept because the involvement of the neighboring structures means that hypothalamic damage and blindness would be inevitable should this be aggressively pursued. ${ }^{28,40}$

Neurosurgery for Secondary Effects of the Tumor or Treatment. Neurosurgery may be required to treat hydrocephalus, to treat the vascular complications of treatment (such as moyamoya disease) and potentially to decompress the optic pathways in cases of progressive visual failure due to progressive cystic disease compressing the optic pathways. In patients with hydrocephalus, there is a clear role for surgery to insert a VP shunt. However, this is not always straightforward, as third ventricle tumors can block the foramina of Monro, requiring either bilateral VP shunt placement or endoscopic fenestration of the septum pellucidum and subsequent insertion of a unilateral VP shunt.

Neurosurgery for the Tumor Itself-Biopsy. Historically, there has not been clear consensus over the role of biopsy at the start of therapy, with controversy as to whether biopsy is necessary at all (independent of NF1 status) if the imaging appearances are typical. ${ }^{16,17,26,29,39}$ Histopathological diagnosis can certainly allow an informed discussion of the therapeutic options and prognosis, especially with the need to distinguish between pilocytic astrocytoma and pilomyxoid astrocytoma. It is therefore not unreasonable to consider an initial biopsy if the imaging appearances are atypical and if the biopsy can be performed with a satisfactory degree of safety. The recent consensus statement has added that biopsy may be indicated at the beginning of nonsurgical treatment (chemotherapy, biological therapy, or radiotherapy) after multidisciplinary discussion, in the context of atypical radiological appearances and/or clinical trials involving biological markers even in patients with NF1. ${ }^{46}$ Suitable methods include endoscopic biopsy, stereotactic biopsy, and open biopsy via craniotomy.

Neurosurgery for the Tumor Itself-Tumor Debulking. Neurosurgery to debulk or excise OPHG has been the subject of much debate, particularly with regard to the timing of surgery. ${ }^{17-19,22,24,26,29,30,34,35,39-42,47}$ These tumors are rare, and it is clear that surgeons need appropriate expertise to safely plan and perform this surgery.

There are many facets to the discussions surrounding the role of debulking surgery. We consider it vital to take into account the tumor size and anatomical location in 3 planes of imaging, as well as the patient's symptoms, whenever surgical debulking of the tumor is being deliberated. The dangers of aggressive neurosurgical tumor excision have been highlighted in other series, with high rates of surgery-related complications, including hypothalamic damage and endocrine deficit. ${ }^{28,40}$ In this series, the neurosurgeon aimed to limit the operative risks by leaving a rim of tumor around the base, performing a predominantly central debulking of the exophytic component of the tumor. Through this approach, none of our surgical cohort of patients showed signs of hypothalamic or visual injury as a direct consequence of surgery.

Tumor growing into the third ventricle is usually an exophytic central tumor component, with the hypothalamic tissue at the base and sides. In these circumstances, the authors' preferred approach to surgery is a midline transcallosal, interforniceal approach, to enter the third ventricle and visualize this exophytic tumor component within the third ventricle. By approaching the tumor in this way, the hypothalamic tissue can be preserved around the base where it blends laterally with the hypothalamus 
avoiding significant hypothalamic dysfunction. A pterional approach was reserved for patients with a significant lateral exophytic component of tumor.

Many authors accept the role of neurosurgical debulking in the context of progression during or after chemotherapy, but this still varies according to surgical expertise and anticipated perioperative risk. Primary neurosurgical tumor debulking (as the main treatment option) is a strategy that is not widely used, largely due to the perceived risks of surgery. Further complicating this debate is uncertainty over whether larger-volume tumor resections improve rates of tumor control. Steinbok et al. found no improvement in control rates, but Wishoff et al. and Hoffmann et al. found that the resection rate did improve tumor control. ${ }^{20,39,40,47}$ Overall, our experience confirms that primary upfront surgery can be both safe and effective. In this series, surgery alone was successful at controlling tumor for 13 of 17 patients (as a primary treatment in 7 of 10 cases [Group 1] and for tumor progression after other treatment in 6 of 7 cases [Group 3]). Disease control was achieved in all $4(100 \%)$ of the patients treated concurrently with surgery and chemotherapy (Group $2)$; this percentage is larger than what has been achieved in many other series, ${ }^{2,11,22,26,29,40}$ although the numbers are clearly small. Spontaneous regression of OPHG following debulking surgery without subsequent adjuvant therapy has been previously reported..$^{20,28,48,50}$ There are also series reporting stabilization of tumor after substantial debulking procedures, without worsening of preoperative deficits. ${ }^{26,29}$ In the patients who were treated surgically in this series, we observed both tumor regression and stabilization of residual disease (Fig. 3).

We consider that there is a definite role for primary tumor debulking for carefully selected patients. Our experience confirms that surgery can successfully control tumor growth, and also that it can be safe. We advocate an aim of limited tailored neurosurgical tumor debulking for selected patients, without aiming for total resection, so that the risk of damage to the surrounding structures is minimized. With this approach we feel we can achieve the optimal balance between tumor debulking, surgeryrelated risk, and surgery-related morbidity. ${ }^{40}$

Surgical Approach. In addition to discussions over the role of surgery, the route employed for surgical access and resection also needs to be considered. The 2 main approaches used for patients in this series were the midline transcallosal approach and the pterional approach. The choice of approach will ultimately be dictated by the primary exophytic component of the tumor and/or cystic extensions.

The midline transcallosal approach has the potential to be safe and extremely effective, particularly where there is an exophytic tumor component largely confined to the third ventricle. As the tumor grows inside the third ventricle, it pushes the hypothalamic tissue laterally. A midline transcallosal approach allows the surgeon to remove the central aspects of the tumor, sparing the hypothalamus and preserving its function. ${ }^{26}$ Such an approach also facilitates the reopening of the CSF pathways and can obviate the need for a VP shunt for treatment of hy- drocephalus. In our institution we have found this to be a safe approach with a low morbidity rate when used for carefully selected cases.

The pterional approach is of particular value for patients whose tumors have significant lateral extension or in whom the optic nerve(s) or chiasm need to be directly decompressed by removal of compressive exophytic and cystic components. ${ }^{26}$ In practice, the role of surgery to improve vision is unproven, unless it is being used to control critically raised intracranial pressure or clear compression of the optic nerves by a cyst.

In addition the pterional approach can allow access to de-roof the optic canal, although we have not needed to do this in any patients in this series. There are, however, potential dangers of this approach, including damage to the perforating branches of the anterior and middle cerebral arteries and damage to the hypothalamus itself. In patients with large tumors, the anatomy can be very distorted, and it can be difficult to define where the hypothalamus and/or optic tracts are expected to be. This will increase the risk of surgical morbidity, and careful consideration is required when planning the approach in such cases. The extent of resection through this approach will often be less than through the midline transcallosal route. This has been the case in our experience, where we have limited the use of the pterional approach to the control of tumor cysts or lateral debulking of exophytic temporal lobe tumor components.

\section{Neurosurgical Advances}

Over the past 20 years, there have been significant improvements in preoperative imaging quality. As a result, the neurosurgeon can have a better understanding of the tumor location and anatomical relations, and thus have an improved appreciation of the potential benefits and risks of different surgical approaches. As high-field advanced MRI scanners become increasingly commonplace, advanced imaging and tractography should add valuable information to the preoperative planning data available. ${ }^{37}$ In particular, identifying the location of the optic pathways inside large tumors would be extremely valuable when planning a surgical approach.

Intraoperative neuronavigation has been a vitally important development. ${ }^{34}$ Different systems are available for use, and they are becoming increasingly technologically advanced in what they can offer the neurosurgeon. Regular use of this tool can also help improve the safety and accuracy of surgery. There are limitations to intraoperative neuronavigation, however. The navigation scans are obtained preoperatively and cannot be readily updated during surgery. Therefore, over the course of an operation, the accuracy of navigation can deteriorate due to tumor volume resection, brain shift, and CSF loss.

The newest developments are coming with the advent of intraoperative MRI. ${ }^{1,349}$ This new tool, used in our institution since 2010, may facilitate safer tumor removal and help to reduce the need for second-look operations for unexpected tumor residuum-achieving the goals of surgery more safely. This, of course, needs to be evaluated in a prospective study, which has been commenced in our institution and will be reported on in the future. 


\section{J. Goodden et al.}

\section{Outcome}

Vision. The assessment of visual function, particularly in younger children can be particularly challenging. A further challenge that we encountered was that the retrospective analysis of visual outcomes is difficult, with results not always clear (or even being missing) in the patient notes. At presentation, children should be seen by a pediatric ophthalmologist and have a thorough examination including assessment of visual acuity, visual fields, color vision, and the fundus. Due to the tumor location, many children present with visual problems or develop visual deficits during treatment, so it is important that they are reassessed on a regular basis., ${ }^{4,43}$ In younger children in particular, there can be quite marked deficits in visual function by the time the diagnosis of OPHG is made - their outward function can often appear intact due to a high level of compensation for visual deficit. ${ }^{43}$ Poor visual function at presentation usually cannot be reversed and is a predictor of poor outcome-the final visual outcome appears to be most related to the patient's visual performance at the time of initial presentation.,42,43 The tailored approach to tumor management used for these patients has resulted in satisfactory rates of visual preservation. ${ }^{4,24,43}$

In general terms, neurosurgical treatment of the tumor itself is rarely employed for children whose vision is actively deteriorating, often because of the complex anatomy and relation to the tumor. In some specific circumstances, neurosurgical operations can be of use-for example where there is direct compression of the optic apparatus by tumor (solid or cystic) or where there is raised intracranial pressure (for example, in patients with giant tumors requiring debulking or hydrocephalus requiring a shunt). ${ }^{44}$

Radiotherapy has been shown to both preserve and improve vision for some patients; ${ }^{4,5,11}$ however, its longterm effects have made treating teams reluctant to employ it now that chemotherapy and surgery have also been shown to be effective., , $5,26,29^{2}$

Endocrine. Endocrine problems either at presentation or developing during treatment are a significant problem for children with OPHG, with a wide variety reported in the literature. ${ }^{21}$ Close collaboration with an endocrine team from the time of first presentation, continuing throughout the treatment period, is clearly important. The rates of new or progressive endocrine deficit over the course of treatment are up to $64 \% .^{5,9,11,14,26}$ It is clear that endocrine function is impaired initially by the tumor itself but is further impaired by the variety of treatment regimens employed to control the tumor. In this series, a mixture of precocious puberty and pituitary hormone deficiencies were evident at first presentation, before any treatment had been undertaken.

Over the treatment period, the number of patients with endocrinopathy increased. Changes in BMI standard scores during the period of observation were also highly variable. Some patients, obese at presentation, significantly improved their BMI to within the normal or overweight range at the most recent assessment. A small- er number of patients gained weight. The regulation of BMI in this heterogeneous cohort of patients is complex, and precise conclusions as to why weight was gained or lost cannot be drawn. We could not find any evidence of neurosurgery-related hypothalamic deficit other than diabetes insipidus in our series.

Differentiating between endocrinopathy due to tumor progression versus complications of treatment is difficult if not impossible-it is clear that significant endocrinopathy would develop in patients even if they were not treated..$^{9,14,21,42}$ There are some suggestions that treatment-associated endocrinopathy is more significant with radiotherapy than chemotherapy or surgery, although not all authors agree..$^{9,21}$

For this cohort of patients, we plan to undertake a comprehensive cross-sectional neuropsychological and functional outcome study in the future.

These observations highlight the importance of close collaboration between health care professionals to optimize patient outcomes through the active management of abnormalities of weight gain, growth, and puberty.

\section{Conclusions}

This study presents a mixed series of cases involving patients with Dodge type $\mathrm{B}$ and $\mathrm{C}$ tumors, (modified Dodge types 2, 3, and 4), with almost half requiring observation only. As expected, patients with NF1 were found to have a more benign form of disease, with fewer patients in the NF1 cohort requiring treatment and lower levels of morbidity developing during the time-course of their treatment, as compared with the non-NF1 cohort.

Primary surgical debulking alone (without adjuvant therapy) can be effective at controlling tumor growth and preserving function, without significant levels of postoperative morbidity. For young, high-risk patients, combining primary surgical debulking with concurrent chemotherapy has also been successful. With careful preoperative planning and goal setting, neurosurgery via a midline transcallosal approach can safely debulk the exophytic central component of third ventricle tumors while preserving hypothalamic and visual function. Surgery can also safely decompress the visual pathways with good success. Intraoperative MRI may help further improve both the volume and the safety of resection, but evaluation is still needed.

Optic pathway/hypothalamic glioma represents a long-term disease, with a prolonged patient journey. We observed that some patients had more aggressive patterns of tumor growth that required multiple interventions to achieve control, raising the question of whether the biological/genetic behavior of this subset of tumors is different from their histologically similar counterparts. Through the adoption of a multidisciplinary tailored approach to management for each case in this series, we were able to achieve good rates of tumor control, and minimize the requirements for subsequent radiotherapy. The best way to care for patients with OPHG is through a cooperative approach involving a skilled and appropriately trained neurosurgeon and oncologist, together with the other members of the neuro-oncology multidisciplinary team. 


\section{Disclosure}

The authors report no conflict of interest concerning the materials or methods used in this study or the findings specified in this paper.

Author contributions to the study and manuscript preparation include the following. Conception and design: Mallucci, Goodden, Pizer. Acquisition of data: Goodden, Williams. Analysis and interpretation of data: Goodden. Drafting the article: Goodden. Critically revising the article: all authors. Reviewed submitted version of manuscript: all authors. Approved the final version of the manuscript on behalf of all authors: Mallucci. Statistical analysis: Goodden. Administrative/technical/material support: Goodden, Williams. Study supervision: Mallucci, Pizer.

\section{References}

1. Abernethy LJ, Avula S, Hughes GM, Wright EJ, Mallucci CL: Intra-operative 3-T MRI for paediatric brain tumours: challenges and perspectives. Pediatr Radiol 42:147-157, 2012

2. Allen JC: Initial management of children with hypothalamic and thalamic tumors and the modifying role of neurofibromatosis-1. Pediatr Neurosurg 32:154-162, 2000

3. Avula S, Mallucci CL, Pizer B, Garlick D, Crooks D, Abernethy LJ: Intraoperative 3-Tesla MRI in the management of paediatric cranial tumours-initial experience. Pediatr Radiol 42:158-167, 2012

4. Campagna M, Opocher E, Viscardi E, Calderone M, Severino SM, Cermakova I, et al: Optic pathway glioma: long-term visual outcome in children without neurofibromatosis type-1. Pediatr Blood Cancer 55:1083-1088, 2010

5. Cappelli C, Grill J, Raquin M, Pierre-Kahn A, Lellouch-Tubiana A, Terrier-Lacombe MJ, et al: Long-term follow up of 69 patients treated for optic pathway tumours before the chemotherapy era. Arch Dis Child 79:334-338, 1998

6. Ceppa EP, Bouffet E, Griebel R, Robinson C, Tihan T: The pilomyxoid astrocytoma and its relationship to pilocytic astrocytoma: report of a case and a critical review of the entity. J Neurooncol 81:191-196, 2007

7. Chan MY, Foong AP, Heisey DM, Harkness WF, Hayward RD, Michalski A: Potential prognostic factors of relapse-free survival in childhood optic pathway glioma: a multivariate analysis. Pediatr Neurosurg 29:23-28, 1998

8. Chikai K, Ohnishi A, Kato T, Ikeda J, Sawamura Y, Iwasaki Y, et al: Clinico-pathological features of pilomyxoid astrocytoma of the optic pathway. Acta Neuropathol 108:109-114, 2004

9. Collet-Solberg PF, Sernyak H, Satin-Smith M, Katz LL, Sutton L, Molloy P, et al: Endocrine outcome in long-term survivors of low-grade hypothalamic/chiasmatic glioma. Clin Endocrinol (Oxf) 47:79-85, 1997

10. Desai SS, Paulino AC, Mai WY, Teh BS: Radiation-induced moyamoya syndrome. Int J Radiat Oncol Biol Phys 65: 1222-1227, 2006

11. Erkal HS, Serin M, Cakmak A: Management of optic pathway and chiasmatic-hypothalamic gliomas in children with radiation therapy. Radiother Oncol 45:11-15, 1997

12. Fernandez C, Figarella-Branger D, Girard N, Bouvier-Labit C, Gouvernet J, Paz Paredes A, et al: Pilocytic astrocytomas in children: prognostic factors - a retrospective study of 80 cases. Neurosurgery 53:544-555, 2003

13. Fisher PG, Tihan T, Goldthwaite PT, Wharam MD, Carson BS, Weingart JD, et al: Outcome analysis of childhood low-grade astrocytomas. Pediatr Blood Cancer 51:245-250, 2008

14. Fouladi M, Wallace D, Langston JW, Mulhern R, Rose SR, Gajjar A, et al: Survival and functional outcome of children with hypothalamic/chiasmatic tumors. Cancer 97:1084-1092, 2003

15. Freeman JV, Cole TJ, Chinn S, Jones PR, White EM, Preece MA: Cross sectional stature and weight reference curves for the UK, 1990. Arch Dis Child 73:17-24, 1995

16. Gajjar A, Sanford RA, Heideman R, Jenkins JJ, Walter A, Li
Y, et al: Low-grade astrocytoma: a decade of experience at St. Jude Children's Research Hospital. J Clin Oncol 15:27922799, 1997

17. Garvey M, Packer RJ: An integrated approach to the treatment of chiasmatic-hypothalamic gliomas. J Neurooncol 28:167183, 1996

18. Grill J, Laithier V, Rodriguez D, Raquin MA, Pierre-Kahn A, Kalifa C: When do children with optic pathway tumours need treatment? An oncological perspective in 106 patients treated in a single centre. Eur J Pediatr 159:692-696, 2000

19. Hoffman HJ, Humphreys RP, Drake JM, Rutka JT, Becker LE, Jenkin D, et al: Optic pathway/hypothalamic gliomas: a dilemma in management. Pediatr Neurosurg 19:186-195, 1993

20. Hoffman HJ, Soloniuk DS, Humphreys RP, Drake JM, Becker LE, De Lima BO, et al: Management and outcome of lowgrade astrocytomas of the midline in children: a retrospective review. Neurosurgery 33:964-971, 1993

21. Jahraus CD, Tarbell NJ: Optic pathway gliomas. Pediatr Blood Cancer 46:586-596, 2006

22. Jaing TH, Lin KL, Tsay PK, Hsueh C, Hung PC, Wu CT, et al: Treatment of optic pathway hypothalamic gliomas in childhood: experience with 18 consecutive cases. J Pediatr Hematol Oncol 30:222-224, 2008

23. Janss AJ, Grundy R, Cnaan A, Savino PJ, Packer RJ, Zackai $\mathrm{EH}$, et al: Optic pathway and hypothalamic/chiasmatic gliomas in children younger than age 5 years with a 6-year followup. Cancer 75:1051-1059, 1995

24. Lee AG: Neuroophthalmological management of optic pathway gliomas. Neurosurg Focus 23(5):E1, 2007

25. Louis DN, Ohgaki H, Wiestler OD, Cavenee WK, Burger PC, Jouvet A, et al: The 2007 WHO classification of tumours of the central nervous system. Acta Neuropathol 114:97-109, 2007

26. Massimi L, Tufo T, Di Rocco C: Management of optic-hypothalamic gliomas in children: still a challenging problem. Expert Rev Anticancer Ther 7:1591-1610, 2007

27. McLone DG: Perspectives on thalamic and hypothalamic tumors of childhood. Pediatr Neurosurg 32:113, 2000

28. Medlock MD, Scott RM: Optic chiasm astrocytomas of childhood. 2. Surgical management. Pediatr Neurosurg 27:129136, 1997

29. Nicolin G, Parkin P, Mabbott D, Hargrave D, Bartels U, Tabori U, et al: Natural history and outcome of optic pathway gliomas in children. Pediatr Blood Cancer 53:1231-1237, 2009

30. Nishio S, Takeshita I, Fujiwara S, Fukui M: Optico-hypothalamic glioma: an analysis of 16 cases. Childs Nerv Syst 9:334-338, 1993

31. Opocher E, Kremer LCM, Da Dalt L, van de Wetering MD, Viscardi E, Caron HN, et al: Prognostic factors for progression of childhood optic pathway glioma: a systematic review. Eur J Cancer 42:1807-1816, 2006

32. Packer RJ, Ater J, Allen J, Phillips PL, Geyer R, Nicholson HS, et al: Carboplatin and vincristine chemotherapy for children with newly diagnosed progressive low-grade gliomas. J Neurosurg 86:747-754, 1997

33. Packer RJ, Lange B, Ater J, Nicholson HS, Allen J, Walker $\mathrm{R}$, et al: Carboplatin and vincristine for recurrent and newly diagnosed low-grade gliomas of childhood. J Clin Oncol 11: 850-856, 1993

34. Rutka JT, Kuo JS: Pediatric surgical neuro-oncology: current best care practices and strategies. J Neurooncol 69:139-150, 2004

35. Sawamura Y, Kamada K, Kamoshima Y, Yamaguchi S, Tajima T, Tsubaki J, et al: Role of surgery for optic pathway/hypothalamic astrocytomas in children. Neuro Oncol 10:725-733, 2008

36. Serdaroğlu A, Simşek F, Gücüyener K, Oğuz A, Karadeniz C, Balibey M: Moyamoya syndrome after radiation therapy for optic pathway glioma: case report. J Child Neurol 15:765-767, 2000 


\section{J. Goodden et al.}

37. Shofty B, Weizman L, Joskowicz L, Constantini S, Kesler A, Ben-Bashat D, et al: MRI internal segmentation of optic pathway gliomas: clinical implementation of a novel algorithm. Childs Nerv Syst 27:1265-1272, 2011

38. Silva MM, Goldman S, Keating G, Marymont MA, Kalapurakal J, Tomita T: Optic pathway hypothalamic gliomas in children under three years of age: the role of chemotherapy. Pediatr Neurosurg 33:151-158, 2000

39. Steinbok P: Optic pathway tumors in children. J Chin Med Assoc 66:4-12, 2003

40. Steinbok P, Hentschel S, Almqvist P, Cochrane DD, Poskitt K: Management of optic chiasmatic/hypothalamic astrocytomas in children. Can J Neurol Sci 29:132-138, 2002

41. Stokland T, Liu JF, Ironside JW, Ellison DW, Taylor R, Robinson KJ, et al: A multivariate analysis of factors determining tumor progression in childhood low-grade glioma: a population-based cohort study (CCLG CNS9702). Neuro Oncol 12: $1257-1268,2010$

42. Sutton LN, Molloy PT, Sernyak H, Goldwein J, Phillips PL, Rorke LB, et al: Long-term outcome of hypothalamic/chiasmatic astrocytomas in children treated with conservative surgery. J Neurosurg 83:583-589, 1995

43. Taylor T, Jaspan T, Milano G, Gregson R, Parker T, Ritzmann $\mathrm{T}$, et al: Radiological classification of optic pathway gliomas: experience of a modified functional classification system. $\mathbf{B r}$ J Radiol 81:761-766, 2008

44. Tong Z, Wanibuchi M, Uede T, Tanabe S, Hashi K: Significant improvement of visual functions after removal of an intracranial giant optic nerve glioma revealing exophytic growth: case report. Neurosurgery 58:E792, 2006

45. Ullrich NJ, Robertson R, Kinnamon DD, Scott RM, Kieran
MW, Turner CD, et al: Moyamoya following cranial irradiation for primary brain tumors in children. Neurology 68:932938, 2007

46. Walker DA, Liu J, Kieran M, Jabado N, Picton S, Packer R, et al: A multi-disciplinary consensus statement concerning surgical approaches to low-grade, high-grade astrocytomas and diffuse intrinsic pontine gliomas in childhood (CPN Paris 2011) using the Delphi method. Neuro Oncol 15:462-468, 2013

47. Wisoff JH, Abbott R, Epstein F: Surgical management of exophytic chiasmatic-hypothalamic tumors of childhood. J Neurosurg 73:661-667, 1990

48. Yoshikawa G, Nagata K, Kawamoto S, Tsutsumi K: Remarkable regression of optic glioma in an infant. Case illustration. J Neurosurg 98:1134, 2003

49. Yousaf J, Avula S, Abernethy LJ, Mallucci CL: Importance of intraoperative magnetic resonance imaging for pediatric brain tumor surgery. Surg Neurol Int 3 (Suppl 2):S65-S72, 2012

50. Žižka J, Eliás P, Jakubec J: Spontaneous regression of lowgrade astrocytomas: an underrecognized condition? Eur Radiol 11:2638-2640, 2001

Manuscript submitted November 11, 2012.

Accepted August 29, 2013.

Please include this information when citing this paper: published online October 18, 2013; DOI: 10.3171/2013.8.PEDS12546.

Address correspondence to: Conor Mallucci, F.R.C.S.(SN), Alder Hey Children's NHS Foundation Trust, Eaton Rd., Liverpool L12 2AP, United Kingdom. email: conor.mallucci@alderhey.nhs.uk. 